\title{
Caregivers described how an Alzheimer's disease respite programme gave them time to attend to their own needs
}

\author{
Perry J, Bontinen K. Evaluation of a weekend respite program for persons with Alzheimer Disease. Can J Nurs Res 2001 \\ Jun;33:81-95.

\section{QUESTION: In caregivers for family members with Alzheimer's disease (AD), what are their experiences of a respite programme?}

Source of funding:

Vancouver-Richmond

branch of the Victorian

Order of Nurses

For correspondence: $\mathrm{Dr}$

J Perry, School of

Nursing, University of

British Columbia,

T201-2211 Wesbrook

Mall, Vancouver, $B C$

V6T 2B5, Canada.
Design

Qualitative study using semistructured interviews.

\section{Setting}

An overnight weekend respite programme in Vancouver, British Columbia, Canada.

\section{Participants}

19 caregivers (11 caregivers were $28-80$ years of age, median age $65 \mathrm{y}$ ) who used the respite service for family members who had AD. Participants were wives $(n=8)$, daughters $(n=3)$, sons $(n=3)$, husbands $(n=2)$, sisters $(\mathrm{n}=2)$, and 1 granddaughter.

\section{Methods}

Participants were interviewed by telephone. Questions included "How did you spend the time while [your family member] was attending the programme?" and "How do you think [your family member] experienced the programme?" Interviews were audiotaped and transcribed. Content analysis was used to identify themes and ideas. Constant comparative analysis was used until consensus was reached on categories and themes.

\section{Main findings}

3 categories were identified.(1) Caregiver self care: 3 themes described how caregivers used strategies to promote their physical, social, and emotional wellbeing. (a) Maintaining family and social relationships: using the time to connect with family and friends. (b) Maintaining health: some caregivers used the time to look after their own health. Respite was used when they were sick or needed to attend to their own psychosocial needs. (c) Catching up on sleep and rest: this theme overlapped with maintaining health. Exhausted caregivers used the time to rest and catch up on sleep. (2) Relief for the caregiver: caregivers appreciated the feeling of being temporarily free from stress and worry. They felt relief because they knew their family member was safe and comfortable. (3) Safety and comfort of the family member: caregivers valued the opportunities for socialising, meaningful interaction, and sensory stimulation that the respite programme offered their family member. They used the criteria of emotional states and physical appearance to evaluate the family member's safety and comfort levels. They also noted how the family member viewed the experience. Caregivers reported that it was difficult to get the client packed up, transported, and settled in the facility, but some felt that the benefit was worth the trouble.

\section{Conclusions}

People who cared for a family member with Alzheimer's disease found a respite programme provided them with time to attend to their own needs, gave them temporary relief from stress and worry, and allowed them to feel that their family member was safe and comfortable.

\section{COMMENTARY}

Research on the effectiveness of respite services for caregivers of family members with $\mathrm{AD}$ has yielded inconsistent results ${ }^{1}$ and has not provided a definitive approach for the conceptualisation of future studies. Despite these facts, clinicians often recommend respite services as an important thrust for the care of the family caregiver, ${ }^{2}$ and consumer groups continue to lobby for a broad range of respite options.

Perry and Bontinen's study has added to the scientific literature by showing what many clinicians hear anecdotally-that caregivers need to be assured that quality care is being given to their relatives before they can experience the temporary relief from worry and stress that the respite intends to provide. They found that the caregivers' judgements about the quality of the care extended beyond process issues (eg, whether the relatives received sufficient sensory stimulation during respite) to include outcomes of care (eg, their relatives' emotional state after respite). This finding highlights the caregivers' level of commitment to their relatives.

The strengths of this study are the inclusion of caregiver representatives in the decision making about the study methods, the use of general open ended questions that did not direct the participants to address the stated programme goals, the clear description of the data analysis, and the safeguard of having clinically oriented staff review the codes and themes for credibility before final consolidations of the findings. The transferability of the findings to other settings would have been enhanced by including more demographic and related data on both the caregivers and their relatives. These findings are limited to overnight respite services and only to programme users.

As indicated by Perry and Bontinen, the finding that temporary relief from worry seems to be related to the caregivers' knowledge that their family members are safe and well suggests that a more family centred model should be considered in future research. From clinical and healthcare planning perspectives, this finding reinforces the importance of both applying explicit family centred models to respite programmes and incorporating this content in programme evaluation strategies. More specifically, discussions about family caregivers' expectations and judgments about the quality of care received by their relatives should be part of ongoing respite care.

E Ann Mohide, RN, MHSc, MSc McMaster University

Hamilton

Ontario Canada

1 Doody RS, Stevens JC, Beck C, et al. Practice parameter: management of dementia (an evidence-based review). Report of the Quality Standards Subcommittee of the American Academy of Neurology. Neurology 2001;56:1154-66.

2 Ham R. Evolving standards in patient and caregiver support. Alzheimer Dis Assoc Disord 1999;13(Suppl 2):S27-35. 